\title{
Multilevel Channel Conflicts with Robust Addressing Protocol in Mobile Ad Hoc Networks
}

\author{
Shweta A Mane ${ }^{1}$, Sanjay S Pawar \\ ${ }^{1}$ M. E E \& TC, Bharati Vidyapeet’s College of Engg, Kolhapur, Shivaji University, Kolhapur, Maharashtra, India \\ ${ }^{2}$ Assistant Professor in E \& TC, Bharati Vidyapeet's College of Engg, Kolhapur, Shivaji University, Kolhapur, Maharashtra, India
}

\begin{abstract}
Ad Hoc networks are the networks that have mobile nodes which communicate with each other through wireless medium without any fixed infrastructure. MANET is a type of ad hoc network that has no centralized control and consists of mobile platforms which are free to move arbitrarily. In mobile ad hoc network, the nodes are mobile and inter-node connectivity may change frequently during normal operation. The MANET has a problem of sending the number of multilevel channels to one network to another network. Also, address assignment for each node in the MANET is a key challenge due to the lack of infrastructure. Finally this problem makes addressing collisions and multilevel channel conflicts. To overcome the address collisions and multilevel channel conflicts, we propose the tumbling multilevel channel conflicts and Filter based addressing protocol. Channel allowance is an elementary affair of resource activity that aggregates the comprehension and extent of attendants. As dormant channel apportionment misses the heuristic applications to allot the channels to the cages headed channel assignment behaves beneficially inferior leaden traffic. Address assignment is a key challenge in ad hoc networks due to the lack of infrastructure. Autonomous addressing protocols require a distributed and self-managed mechanism to avoid address collisions in a dynamic network with fading channels, frequent partitions, and joining/leaving nodes. We propose and analyze a lightweight protocol that configures mobile ad hoc nodes based on a distributed address database stored in filters that reduces the control load and makes the proposal robust to packet losses and network partitions. We evaluate the performance of our protocol, considering joining nodes, partition merging events, and network initialization.
\end{abstract}

Keywords: Mobile Ad Hoc Networks, Channel conflicts, frequency reuse, auto-configuration protocol, address collision, network management.

\section{Introduction}

MANET is a type of ad hoc network that can change locations and configure itself on the fly. Because MANETS are mobile, they use wireless connections to connect to various networks. This is in contrast with the topology of the existing Internet, where the router topology is essentially static. The MANET has a problem of sending the number of multilevel channels to one network to another network. Also, address assignment for each node in the MANET is a key challenge due to the lack of infrastructure. Finally this problem makes addressing collisions and multilevel channel conflicts. To overcome the address collisions and multilevel channel conflicts, we propose the tumbling multilevel channel conflicts and Filter based addressing protocol [1][2]. First method is tumbling multilevel channel conflicts. It depends on three steps: calculates number of loops in the network, using a multi channel sorting algorithm, and then apply an algorithm for 3C approach for allocating the conflict free channels. These three algorithms calculate the number of channels and assign the demand value. They are sorting the channels by the demand value by using a multilevel channel sorting algorithm and allocating to send the channels by using the $3 \mathrm{C}$ algorithm [2]. Second proposed method is Filter based addressing protocol. This protocol aims to dynamically auto configure network addresses, resolving collisions with a low control load, even in joining or merging events. It uses a distributed compact filter to represent the current set of allocated addresses. By simulation it is proved that the proposed method reduces the address collisions and multi level channel conflicts in Mobile Ad Hoc Networks [1].

\section{Relevance}

Before implementing addressing protocol for node auto configuration we see that how to reduce the Multilevel Channel Conflicts in Mobile Ad Hoc Networks. Channel allowance is an elementary affair of resource activity that aggregates the comprehension and extent of attendants. As dormant channel apportionment misses the heuristic applications to allot the channels to the cages, headed channel assignment behaves beneficially inferior leaden traffic. Assiduous channel allowance apprises behaves inferior brightness as well as alleviated traffic [2].

Autonomous addressing protocols require a distributed and self-managed mechanism to avoid address collisions in a dynamic network with fading channels, frequent partitions, and joining/leaving nodes. Address assignment is a key challenge in ad hoc networks due to the lack of infrastructure. We propose and analyze a lightweight protocol that configures mobile ad hoc nodes based on a distributed address database stored in filters that reduces the control load and makes the proposal robust to packet losses and network partitions [1].

We evaluate the performance of addressing protocol, considering joining nodes, partition merging events, and network initialization. Addressing protocol resolves all the address collisions and also reduces the control traffic when compared to previously proposed protocols. The proposed mechanism for node is able to allot the conflict free channels only for four cells clusters architecture and seven cells cluster arrangement and consequences arises that allocated channels are conflict free and based on the compatibility 


\section{International Journal of Science and Research (IJSR) \\ ISSN (Online): 2319-7064}

Index Copernicus Value (2013): 6.14 | Impact Factor (2015): 6.391

matrix which is allocation methodology.

\section{Multilevel Channel Conflicts with Robust Addressing Protocol in MANETs}

\subsection{Multilevel Channel Conflicts in MANETs}

WiMax / UMTS infrastructure is observing for expediency of Mobile Ad Hoc Networks. The core analysis challenge in WiMax is about the anode allowance. Additionally destined to deficient anode affiliate and bulky amplification in mobile consumers, the difficulty of contest free channels allocation dovetails very arrogant. Hence, the main objective of this thesis is to reduce the Multilevel Channel Conflicts in Mobile Ad Hoc Networks [2]. Channel allowance is an elementary affair of resource activity that aggregates the comprehension and extent of attendants. As dormant channel apportionment misses the heuristic applications to allot the channels to the cages. Headed channel assignment behaves beneficially inferior leaden traffic. Assiduous channel allowance apprises behaves inferior brightness as well as alleviated traffic. To advance the benefit of the channel chunk we try an alloyed application for channel allowance in which FCA and DCA apprises coupled will work concomitantly. The results depict that the proposed mechanism is able to allot the conflict free channels to all enclosures according to the constraint of the enclosures. This allocation is able to reduce the conflicts in multi level channels in ad hoc networks.

\section{Cluster Formation}

Frequency reuse is possible only with the help of reuse distance. Reused distance is defined as the distance between the two cells using the same channel. It is denoted by D [20]. There is a close relationship between the reuse distance $\mathrm{D}$, radius of cell $\mathrm{R}$ and the number of cells $\mathrm{N}$ of a cluster i.e

$$
\mathrm{D}=\sqrt{3} \mathrm{~N} \text { R }
$$

So reuse factor will be,

$$
\mathrm{D} / \mathrm{R}=\sqrt{ } \mathrm{3N}
$$

So minimum distance ratio required for clustering for co channel is $\sqrt{3} \mathrm{~N}$. The number of cells, $\mathrm{N}$ per cluster is given by $\mathrm{N}=\mathrm{i}^{2}+\mathrm{ij}+\mathrm{j}^{2}$. Here $\mathrm{i}$ denotes the number of cells along the direction $\mathrm{i}$ from center of a cell and $\mathrm{j}$ denotes number of cells at $60^{\circ}$ to the direction of $\mathrm{i}$ and the value of $\mathrm{N}$ [1] will be $1,3,4,7,9,12,13,16 \ldots$. The size of cluster of 1 is possible but in this concept of reuse is violated because high degree of interference and maximum cell size is possible 100 kilometers. Ideal cell size possible is from 100 meters to 20 kilometers. 100 meters for highly dense areas of metropolitan cities and $20 \mathrm{~km}$ is for urban areas. But in practical cluster size is 7 and most of the service providers are using this size.
4 cells

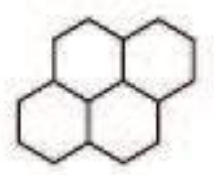

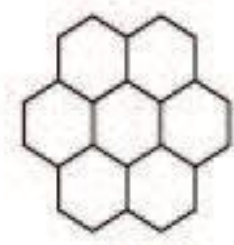

7 cells
Figure 1: 4 cells and 7 cells cluster

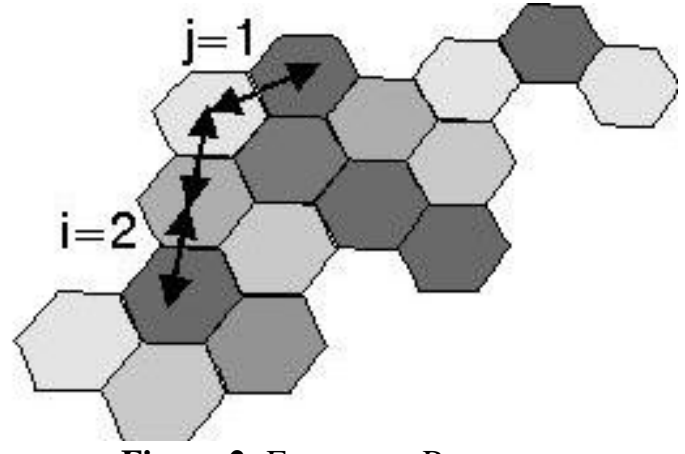

Figure 2: Frequency Reuse

Frequency Reuse is the core concept of cellular mobile radio. Users in different geographical areas (in different cells) may simultaneously use the same frequency. Frequency reuse drastically increases user capacity and spectrum efficiency. Frequency reuse causes mutual interference (trade off link quality versus subscriber capacity).

\section{Co-channel Interference Ratio-}

It is the ratio of co-channel cells and one of the important parameters for simulating the performance of the system. Design is only acceptable if this value is more than 17 . If value is in between 15 to 17 then it is acceptable in some extent. But if value is less than 15, then design of the system is not acceptable and it shows that there is a high degree of interference between the co-channel cells. The value of CCIR is generated on the basis of the formulae given below. And we can also generate the CCIR formula for different location area of the cells [2].

$$
\underset{i=1}{m} / I=1\left(\left(\Sigma\left(D_{K} / R\right)^{\wedge}-\gamma\right)\right.
$$

where,

$\mathrm{m}$ denotes the maximum number of co-channel cells;

$\gamma$ is propagation path loss and its value is varied between 2 to 5 ;

$\mathrm{D}_{\mathrm{K}}$ is the distance from the $\mathrm{k}^{\text {th }}$ cell;

$\mathrm{R}$ is the radius of the cell;

$\mathrm{C} / \mathrm{I}$ is the co-channel interference ratio.

\subsection{Node Auto-configuration in MANETs}

The TCP/IP protocol allows the different nodes from the network to communicate. This is done by associating a distinct IP address to each node of the same network. There is a server or node which assigns these IP addresses in wired or wireless networks with an infrastructure. But in Mobile ad-hoc networks, some protocol is needed to perform this function due to lack of centralized entity. This protocol performs the network configuration in a dynamic and automatic way, which utilizes all the nodes of the network or a part of it, as if they were servers that manage IP addresses. Due to dynamic technology of mobile ad hoc networks constant movement of the nodes that can join and leave the network frequently and simultaneously. Auto configuration protocols are faced with various problems that guarantee the uniqueness of IP addresses and allows network partitioning and merging. It is said that, to guarantee the correct 


\section{International Journal of Science and Research (IJSR) \\ ISSN (Online): 2319-7064 \\ Index Copernicus Value (2013): 6.14 | Impact Factor (2015): 6.391}

functioning of the network, the protocols should achieve some objectives. These objectives include assigning unique IP addresses, functioning correctly, fix the problems derived from the loss of messages, allow the multi-hop routing, minimizing the additional packet traffic in the network, verify the existence of competing petitions for an IP address and conduct synchronization [1].

\section{Filter-based Addressing Protocol-}

FAP aims to dynamically auto configure network addresses, resolving collisions with a low control load, even in joining or merging events. To achieve all these objectives, it uses a distributed compact filter to represent the current set of allocated addresses. This filter is present at every node to simplify frequent node joining events and reduce the control overhead required to solve address collisions inherent in random assignments. Moreover, the filter signature which is the hash of the address filter, acts as a partition identifier. The filter signature is an important feature for easily detecting network merging events, in which address conflicts may occur. There is a use of two different filters: the Bloom filter, which is based on hash functions and the Sequence filter, which compresses data based on the address sequence.

\section{Procedures of FAP}

\section{Network Initialization:-}

The network initialization procedure deals with the autoconfiguration of the initial set of nodes. Two different scenarios can happen at the initialization: the joining nodes arrive one after the other with a long enough interval between them, called gradual initialization, or all the nodes arrive at the same time, called abrupt initialization. Most protocols assume the gradual scenario with a large time interval between the arrival of the first and the second joining nodes.

\section{Node Ingress and Network Merging Events}

After the initialization, each node starts broadcasting periodic Hello messages containing its address filter signature. Upon the reception of a Hello, neighbors evaluate whether the signature in the message is the same as its own signature to detect merging events. Only the nodes that have already joined the network are able to send Hello messages, receive a request of a node to join the network, and detect merging events.

\section{Node Departure}

When a node leaves the network, its address should become available for the other nodes. If the departing node is correctly shut down, it floods the network with a notification to remove its address from the address filter. If the departing node does not notify the network, the address remains allocated in the filters, which can make the available addresses scarce with time.

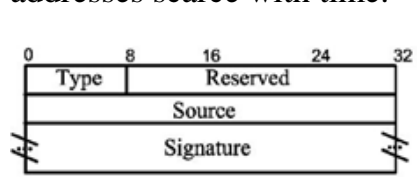

(a)

\begin{tabular}{|l|l|l|}
\hline Type & R & Reserved \\
\hline \multicolumn{3}{|c|}{ Selected address } \\
\hline \multicolumn{3}{|c|}{ Identifier Number } \\
\hline & Signature \\
\hline
\end{tabular}

(b) (c)

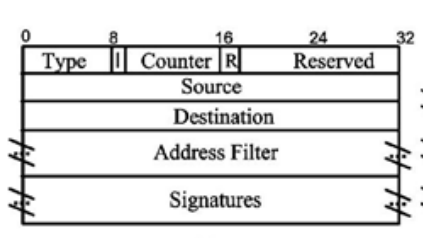

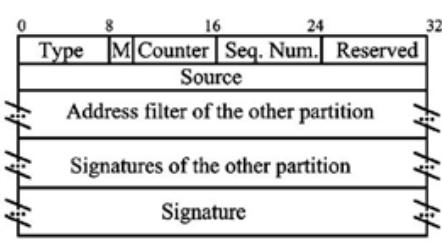

(d)
Figure 3: Messages of FAP for initialization, joining node and partition merging procedures. (a) Hello. (b) AREQ. (c) AF. (d) Partition

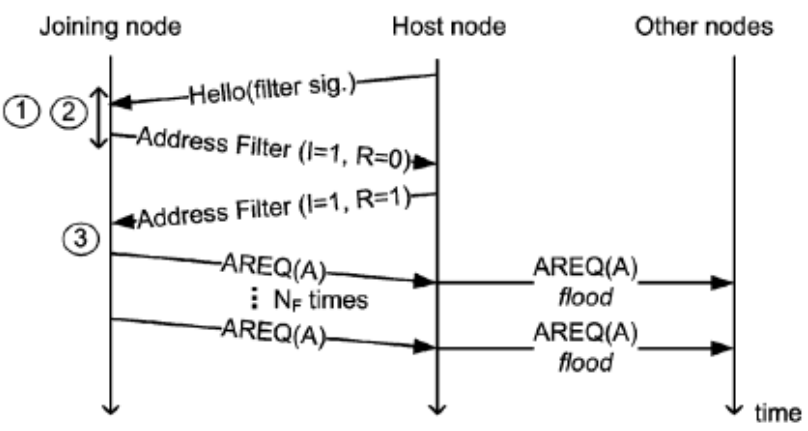

1) Time waiting for hellos $\left(T_{L}\right)$

2) Identify a joining node procedure

3) Store filter and select an available address, such as A

(a)

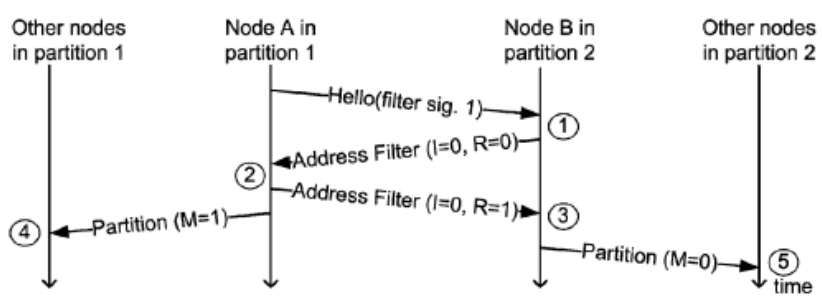

1)Detect a merge event due to a different filter signature in the Hello

2)Merge filters

3)Merge filters and check for collisions because partition 1 is greater than partition 2

4)Merge filters

5)Merge filters and check for collisions because $M=0$ (b)

Figure 4: FAP schemes for node ingress and network merge.

(a) Node ingress.

(b) Partition detection and merge.

\section{Simulation Results}

NS-2 simulator is being used for the proposed system. NS2 is an open-source event-driven simulator designed specifically for research in computer communication networks. Network Simulator (Version 2), widely known as NS2, is simply an event driven simulation tool that has proved useful in studying the dynamic nature of communication networks. Simulation of wired as well as wireless network functions and protocols (e.g., routing algorithms, TCP, UDP) can be done using NS2. In general, NS2 provides users with a way of specifying such network protocols and simulating their corresponding behaviors. 


\section{International Journal of Science and Research (IJSR) \\ ISSN (Online): 2319-7064}

Index Copernicus Value (2013): 6.14 | Impact Factor (2015): 6.391

Table.1: Channel Allocation table

- At time 2.42s

\begin{tabular}{|c|c|}
\hline Cell (CH) & Channels required \\
\hline $1(0)$ & $2,18,13,10,23,28,32$ \\
\hline $2(56)$ & $38,45,47,52$ \\
\hline $3(57)$ & 60 \\
\hline
\end{tabular}

- At time $9.11 \mathrm{~s}$

\begin{tabular}{|c|c|c|}
\hline Cell (CH) & Channels awaiting & Channels alloted \\
\hline $1(0)$ & $3,12,16,7,21,26,31$ & 8,18 \\
\hline $2(56)$ & $40,41,50,51$ & 38 \\
\hline $3(57)$ & $61,67,68,73$ & 71,78 \\
\hline
\end{tabular}

\section{Conclusion and Future Scope}

The proposed scheme offers a simple and light weight mechanism for solving the problem of channel conflicts in multilevel channel case and thus also makes a system robust to auto node configuration to avoid address conflicts in Mobile Ad Hoc Networks. The 3C approach used central pool of frequencies and thus allocates the frequencies as per the demand. FAP protocol fits well for dynamic ad hoc networks with fading channels, frequent partitions and joining/leaving nodes. Thus, the key idea was to use address filters to avoid address collisions, reduce control overhead and decrease the address allocation delay.

Our filter-based protocol increases the protocol robustness to message losses that is actually an important issue for mobile ad hoc networks. The use of the hash of the filter instead of a random number as the partition identifier creates a better representation of the set of nodes. FAP presents smaller delays in the joining node procedure and on network partition merging events than the other proposals, indicating that the proposed protocol is more suitable for very dynamic environments.

\section{References}

[1] Natalia Castro Fernandes, Marcelo Duffles Donato Moreira,and Otta Carlos Muniz Bandeira Duarte, "An Efficient and Robust Addressing Protocol for Node Auto configuration in Ad Hoc Networks", IEEE/ACM Transactions on Networking, 2013. A preliminary issue - Proceedings of the IEEE International Conference on Computer Communications, April 19-25, 2009

[2] Mohammad Abd Elhamid Abbas, Husain Shahnawaz, "Tumbling Multilevel Channel Conflicts in Mobile Ad Hoc Networks", 22-23 Feb, $20133^{\text {rd }}$ IEEE International Advance Computing Conference (IACC)

[3] D. O. Cunha, O. C. M. B. Duarte, and G. Pujolle, "A cooperation aware routing scheme for fast varying fading wireless channels", IEEE Commun. Lett., vol. 12, no. 10, pp. 794-796, Oct. 2008.

[4] N. C. Fernandes, M.D.Moreira, and O. C. M. B. Duarte, "A self-organized mechanism for thwarting malicious access in ad hoc networks", in Proc. 29th IEEE INFOCOM Miniconf., San Diego, CA, Apr. 2010, pp. $1-5$.

[5] N. C. Fernandes, M.D.Moreira, and O. C. M. B. Duarte, "An efficient filter-based addressing protocol for auto configuration of mobile ad hoc networks", in Proc. 28th
IEEE INFOCOM, Rio de Janeiro, Brazil, Apr. 2009, pp. 2464-2472.

[6] C. E. Perkins, E. M. Royers, and S. R. Das, "IP address auto configuration for ad hoc networks", Internet draft, 2000.

[7] Dharma Prakash Agrawal and Qing- An Zeng, "Introduction to Wireless and Mobile System", University of Cincinnati.

[8] A. Gamst and W. Rave, "On frequency assignment in mobile automatic telephone systems", 172 Proceedings of IEEE GLOBECOM'82; Pages 309-315, 1982.

[9] Brent A. Peacock, "Connecting the EDGE: Mobile Ad Hoc networks for Network Centric Warfare", Blue Horizons Paper, Center for Strategy and Technology

[10] K.N.Sivarajan, R.J.McEliece and J.W.Ketchum, "Channel assignment in cellular radio", in Proc. 39th IEEE Veh. Technology. Soc. Conf., pp. 846-850, May 1989.

[11]W.Wang and C.K Rushforth, "An Adaptive LocalSearch Algorithm for the Channel-Assignment Problem (CAP)", IEEE Trans on Veh Technology, vol 45, pp. 459-446, August 1996.

[12] Stephen Toner and Donal O’Mahony, "Self Organising Node Address Management in Ad-hoc Networks", Networks \& Telecommunications Research Group (NTRG), Dublin 2, Ireland

[13]Z. Fan and S. Subramani, "An address autoconfiguration protocol for IPv6 hosts in a mobile ad-hoc network", Comput. Commun., vol. 28, no. 4, pp.339-350, Mar 2005

[14]Kilian Weniger, Martina Zitterbart, “ IPv6 Autoconfiguration in Large Scale Mobile Ad-hoc Networks", Institute of Telematics, University of Karlsruhe, Germany

[15]Chang-Bian Yang et al, "Conflict-free Sorting Algorithms under Single Channel and Multi-Channel Broadcast Communication Models", Advances in Computing and Information, Lecture notes in Computer Science, 1991, Volume 497/1991,350-359

[16] Mr.B.V. Baiju, Mr. Sudhakar Rajendram, "Channel Aware Routing Protocol for MANET", International Journal of Power Control Signal and Computation, Vol.3.No.1. Jan-Mar 2012 ISSN: 0976-268X

[17]Luis Javier Garcia Villalba, Julian Garcia Matesanz, Ana Lucila Sandoval Orozco and Jose Duvan Marquez Diaz, "Auto-Configuration Protocols in Mobile AD Hoc Networks," Sensors 2011 Journal A. Bonnaccorsi, "On the Relationship between Firm Size and Export Intensity," Journal of International Business Studies, XXIII (4), pp. 605-635, 1992. (journal style)

\section{Author Profile}

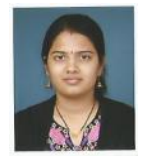

Shweta Mane was born in India and has obtained her B. E degree in Electronics Engineering from Shivaji University, Kolhapur, Maharashtra, India in 2012 Presently, she is a P.G student of E \& TC in Shivaji University, Kolhapur, Maharashtra, India. Prior to that, she had completed her Diploma in Industrial Electronics from Government Polytechnic, Kolhapur. Her current work is related in Mobile Ad Hoc Networks. 